\title{
Evaluación de la generación de vapor de la fábrica de refrescos 23 de Agosto, en Camagüey
}

\author{
Amaury Pérez-Sánchez ${ }^{1^{*}}$, Yenney Fernández Tejeda², Raúl González de la Cruz ${ }^{3^{*}}$ \\ * Universidad de Camagüey Ignacio Agramonte y Loynaz, Facultad de Ciencias Aplicadas a la Industria, Departamento de Química, carretera Circunvalación \\ Norte, km 512/2, e/ camino viejo de Nuevitas y av. Ignacio Agramonte CP 74650, Camagüey, Cuba. \\ * Autor para correspondencia: amauryps@nauta.cu. \\ ** Universidad de Camagüey Ignacio Agramonte y Loynaz, Facultad de Ciencias Aplicadas a la Industria, Departamento de Ingeniería Química, carrera de Inge- \\ niería Química, carretera Circunvalación Norte, km 5ํㅡㄹ, e/ camino Viejo de Nuevitas y av. Ignacio Agramonte, Camagüey, Cuba. CP 74650. \\ * Autor para correspondencia: raul.gonzalez@reduc.edu.cu
}

\section{RESUMEN}

El presente trabajo se llevó a cabo en la fábrica de refrescos 23 de Agosto, con el fin de evaluar la eficiencia energética del generador de vapor, determinar las pérdidas de calor en las tuberías de vapor que se encuentran sin aislamiento, determinar el impacto medioambiental que producen los gases de combustión generados, evaluar el sistema de tratamiento del agua con que se alimenta la caldera (suavizador de agua), así como efectuar un análisis técnico-económico de dos variantes de mejoras, consistentes en 1) precalentamiento del agua con que se alimenta la caldera hasta los $90^{\circ} \mathrm{C}$, y 2) aislamiento de las tuberías de vapor con el aislante lana mineral. El empleo del aislante lana mineral redujo las pérdidas de calor en las tuberías en un $90 \%$, aproximadamente. La eficiencia energética del generador de vapor fue de $86.64 \%$. Se requiere invertir $\$ 523.66$ pesos cubanos (PC) para adquirir e instalar las cantidades necesarias del aislante lana mineral, que se recuperará al cabo de 122 días. El suavizador opera de forma eficiente y los gases contaminantes alcanzan la máxima concentración a los $87.285 \mathrm{~m}$, estando por encima del valor establecido en las normas cubanas. La aplicación de las dos variantes de mejora propuestas trajo consigo un ahorro total anual de PC 9931.92 por el combustible dejado de consumir en el generador de vapor. Se emplearon programas y herramientas computacionales tales como el Contamín, y hojas de cálculo Excel para el procesamiento de los datos adquiridos.

Palabras clave: evaluación, caldera, medioambiente, agua, pérdidas de calor.

Citation: Pérez Sánchez, A. Fernández Tejeda, F. y González de la Cruz, R. (2017). Evaluación de la generación de vapor de la fábrica de refres$\cos 23$ de Agosto, en Camagüey. Mutis 7(1), 7-19, doi: http://dx.doi. org/10.21789/22561498.1198

Received: Julio 11, 2016. Accepted: Marzo 17, 2017. Published on line: Mayo 1, 2017.

Copyright: $\odot 2017$ Pérez-Sánchez, A. Fernández Tejeda, F. y González de la Cruz, R. This is an open-access article, which permits unrestricted use, distributions and reproduction in any me $\neg$ dium, provided the original author and source are credited.

Competing Interests: The authors have no conflict of interest.

\section{Assessment of the steam generation at 23 de Agosto Soda Factory, in Camagüey}

\begin{abstract}
The present work was carried out in the Factory of Sodas 23 de Agosto, with the aim to evaluate the thermal efficiency of the boiler, determine the heat losses in uninsulated steam pipes; determine the environmental impact of flue gases generated; evaluate the boiler's feedwater treatment system (water softener),
\end{abstract}


as well as to make a techno-economic analysis of two improvement alternatives, consistent in: 1) Pre-heating of the feeding water to the boiler up to the 90 ${ }^{\circ} \mathrm{C}$, and 2) Insulation of the steam pipes with mineral wool. The use of the insulating material mineral wool reduced the pipe heat losses in $90 \%$, approximately. The thermal efficiency of the steam generator was $86.64 \%$. It is required to invest $\$ 523.66$ Cuban pesos (CP) to acquire and install the necessary quantities of mineral wool, with a payback period of 122 days. The water softener equipment works efficiently, and the polluting gases reached the maximum concentration at a distance of $87.285 \mathrm{~m}$, which is above the established value by the Cuban standards. The application of the two alternatives of improvement brought about 9931.92 CP of annual total savings due to fuel not consumed in the boiler. Several programs and tools were used to process and analyze the required data, such as the Contamín, and Excel spreadsheets.

Keywords: Assessment, boiler, environment, water, heat losses.

\section{INTRODUCCIÓN}

El vapor de agua es uno de los medios de transmisión de calor de mayor efectividad disponibles hoy en día, y su fácil generación y manejo lo han situado como uno de los servicios auxiliares más difundidos en la industria actual. En los diagnósticos energéticos se han encontrado grandes potenciales de ahorro en la generación y distribución de vapor, que van desde 5 hasta $40 \%$ del consumo de combustible (Colciencias, 2009) (Krishnanunni, Paul, Potti \& Mathew, 2012). La caldera o generador de vapor no es más que un equipo térmico conformado por un recipiente metálico cerrado de forma hermética y presurizado, destinado a producir vapor o calentar agua u otro fluido mediante un proceso isobárico, a partir del suministro de una fuente de energía primaria (generalmente un combustible), que libera ciertas cantidades de calor que luego es aprovechado de manera indirecta por un fluido (generalmente agua) (Guevara, 2013). El tratamiento del agua de alimentación de un generador de vapor es muy importante para asegurar una larga vida útil de este equipo manteniéndolo libre de problemas operacionales, reparaciones de importancia y accidentes, evitándose así inconvenientes por corrosión e incrustaciones (Morales, 2011). El aislamiento térmico se emplea para reducir las pérdidas térmi- cas en sistemas termoenergéticos mediante el cerramiento de los equipos, depósitos, tuberías, etc., los cuales, debido a las demandas térmico-mecánicas o a las elevadas temperaturas a las que normalmente están sujetos, son construidos con materiales metálicos con elevadas conductividades térmicas (Isover, 2012). La evaluación energética de calderas pirotubulares ha sido efectuada con anterioridad por varios autores (Colciencias, 2009) (López, 2009) (Krishnanunni et al., 2012) (Guevara, 2013) (Raut, Kumbhare \& Thakur, 2014) (Meena \& Singh, 2015), empleando diversas metodologías de cálculo disponibles en la actualidad.

La fábrica de refrescos 23 de Agosto se encuentra ubicada en la ciudad de Camagüey, Cuba, y posee una tecnología de procedencia fundamentalmente búlgara, aunque con el transcurso de los años y el deterioro de los equipos y piezas, se ha hecho necesario remodelarla, adquiriendo el equipamiento necesario en otros países, tales como Brasil, Argentina y Rusia. Dados los años de funcionamiento y explotación que lleva esta fábrica, así como también a causa del mantenimiento no adecuado de algunos componentes y equipos del sistema productivo, resulta necesario llevar a cabo una valoración técnico-económica de esta planta con el fin de detectar los problemas de mayor influencia en la eficiencia global del proceso productivo. Con relación a esto, se propuso llevar a cabo, en primera instancia, la evaluación del sistema termoenergético de dicha fábrica, por ser esta área una de las que más deficiencias técnicas presenta y, por consiguiente, una de las de mayor influencia en los costos de producción. Asimismo, se procedió a determinar las pérdidas de calor al ambiente en los tramos de tubería sin aislamiento térmico, la evaluación del sistema de tratamiento de agua que va a la caldera y también la evaluación del impacto medioambiental de los gases de combustión generados.

\section{MATERIALES Y MÉTODOS}

\section{Descripción del área de generación y distribución de vapor}

El área de generación de vapor cuenta con una caldera cilíndrica horizontal del tipo pirotubular, cuyos principales parámetros se muestran en la tabla 1. 
Tabla 1. Principales parámetros del generador de vapor instalado en la fábrica

\begin{tabular}{lll}
\hline Parámetro & Valor & Unidades \\
\hline Generación máxima de vapor & 2500 & $\mathrm{~kg} / \mathrm{h}$ \\
Superficie de calefacción & 76.22 & $\mathrm{~m}^{2}$ \\
Fecha de fabricación & 1983 & - \\
Presión máxima de trabajo autorizada por el fabricante & $13 \mathrm{bar}$ & $\mathrm{bar}$ \\
Presión máxima de trabajo actual & $8 \mathrm{bar}$ & $\mathrm{bar}$ \\
Volumen de agua a nivel máximo admisible de agua & 6.87 & $\mathrm{~m}^{3}$ \\
Volumen de vapor a nivel máximo admisible de agua & 1.58 & $\mathrm{~m}^{3}$ \\
Diámetro del casco & 2050 & $\mathrm{~mm}$ \\
Material & Acero $18 \mathrm{k}$ & - \\
\hline
\end{tabular}

La caldera es adaptable al consumo de tres tipos de combustibles: gas licuado (fundamentalmente para el encendido de la caldera y posterior precalentamiento del combustible), fuel oil (flujo de alimentación: 121 L/h) y diésel (flujo de alimentación: 57 L/h), siendo el fuel oil el principal combustible que utiliza durante su operación normal. Como material aislante tiene lana de vidrio recubierta por una lámina de acero inoxidable. La temperatura de salida de los gases de combustión oscila entre 190 y $260{ }^{\circ} \mathrm{C}$. El vapor de agua generado por la caldera es consumido en tres áreas del proceso: el tanque de mezcla (disolutor), el precalentador del agua y el pasteurizador (véase la fig. 1).

El agua de alimentación de la caldera proviene del acueducto, y es, generalmente, agua tratada que presenta una calidad uniforme. En este caso, el agua que será consumida por la caldera es bombeada hacia el suavizador, que emplea una resina intercambiadora catiónica (Lewatit ${ }^{\circ}$ ) con el objetivo de reducir la dureza total de la misma hasta valores según la norma NRIAL 012, "Aguas industriales-aguas de calderas: especificaciones de calidad" (Minal, 1992). Una vez tratada (suavizada), el agua es enviada hacia un tanque recolector de agua suave, antes de ser dirigida al generador de vapor a una presión de $8 \mathrm{kgf} / \mathrm{cm}^{2}$ por medio de una bomba centrífuga.

\section{Características de las tuberías por donde circula vapor}

En la tabla 2 se exponen los principales datos de los tramos de tuberías por donde circula el vapor de agua en la fábrica, en cuanto a longitudes, diámetros y configuración.

Tabla 2. Datos de los tramos de tubería por donde circula vapor de agua

\begin{tabular}{|c|c|c|c|c|c|c|}
\hline \multirow[t]{2}{*}{ 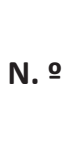 } & \multirow{2}{*}{ Tramo } & \multirow[t]{2}{*}{$\mathrm{T}\left({ }^{\circ} \mathrm{C}\right)$} & \multirow{2}{*}{$\begin{array}{c}\text { Diámetro } \\
\text { interior } \\
(\mathrm{mm})\end{array}$} & \multirow{2}{*}{$\begin{array}{l}\text { Diámetro } \\
\text { exterior } \\
(\mathrm{mm})\end{array}$} & \multicolumn{2}{|c|}{$\begin{array}{l}\text { Longitud } \\
\text { (m) }\end{array}$} \\
\hline & & & & & H & $\mathbf{v}$ \\
\hline 1 & Caldera - calentador de combustible & 158 & 52.5 & 60.3 & 12.36 & 1.55 \\
\hline 2 & Caldera - disolutor & 158 & 77.9 & 88.9 & 17.60 & 1.40 \\
\hline
\end{tabular}

$\mathrm{H}$ : horizontal; V: vertical

En la fig. 1 se muestra el esquema de generación y distribución de vapor (esquema termoenergético) de la fábrica de refrescos 23 de Agosto. 


\section{Metodologías utilizadas para los cálculos}

\section{Metodología para determinar las pérdidas de calor en la red de tuberías de vapor}

Para determinar las pérdidas de calor se pueden emplear varias metodologías de cálculo. La que se propone en este trabajo es un programa Excel basado en metodologías existentes en los textos básicos de transferencia de calor (Isachenko, Osipova \& Sukomel, 1973) (Mijéev \& Mijeeva, 1979) (Pankrátov, 1987) y el folleto Cálculo de las pérdidas de calor y del espesor del aislante (Fonte, Nemiróvich, \& González, 1986). Para utilizar este programa, en principio se realizó un levantamiento hidráulico detallado del sistema de tuberías por donde se transporta el vapor de agua en la fábrica, dividiendo este sistema en tramos (o subtrayectos), para facilitar los cálculos.

\section{ESQUEMA TERMO-ENERGÉTICO \\ Fabrica de Refrescos "23 de Agosto" \\ Camagüey}

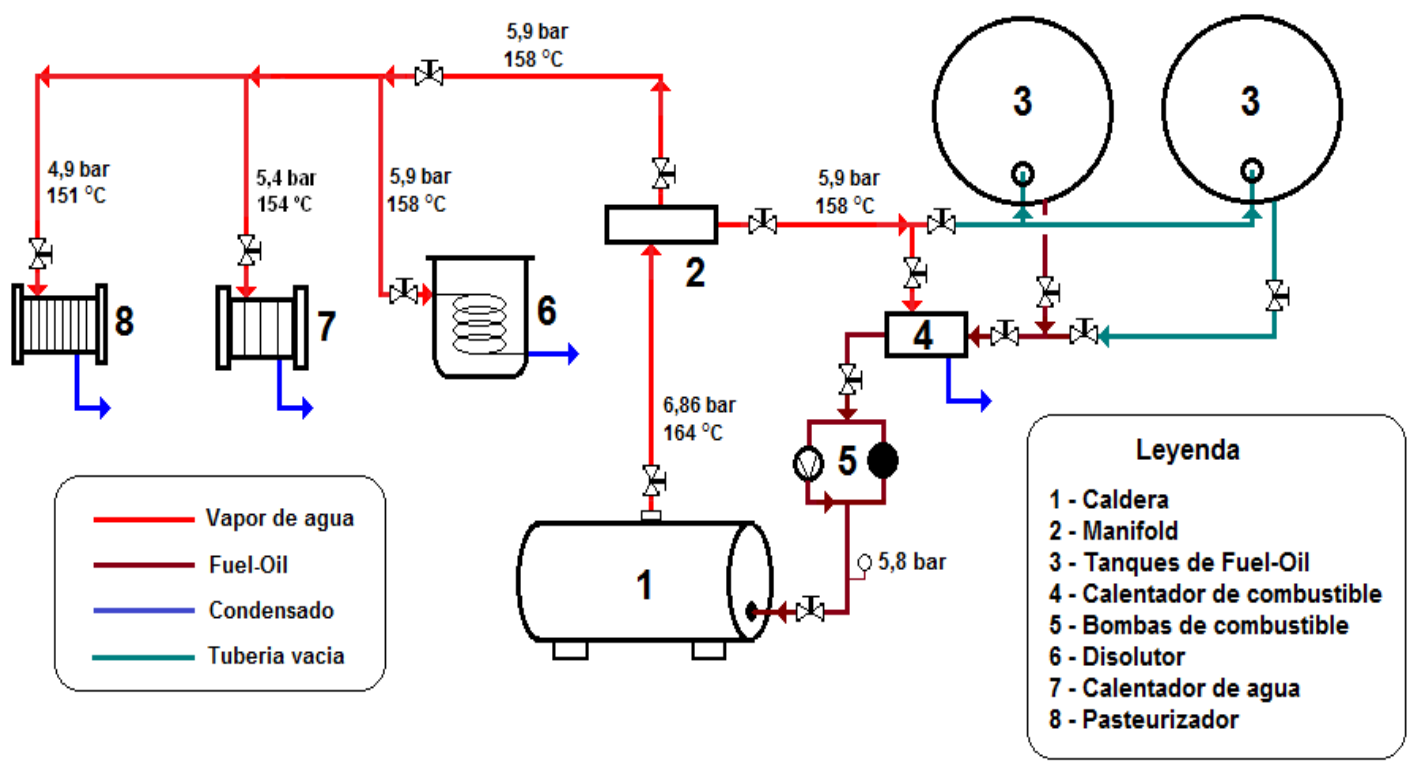

Figura 1. Esquema de generación y distribución de vapor (termoenergético) de la fábrica de refrescos 23 de Agosto

\section{Metodología para determinar el espesor óptimo}

\section{del aislante}

Se propone aislar todas las tuberías por donde circula vapor de agua con lana mineral. En principio se asumirá el diámetro exterior que deberá tener el aislante una vez instalado en la tubería $\left(D_{\text {aisl }}\right)$, así como también la temperatura de la superficie exterior del mismo $\left(T_{\text {ais }}\right)$.

La lana mineral presenta las siguientes propiedades físicas (Calorcol, 2014):

- Conductividad térmica $\left(\lambda_{\text {ais }}\right)=0.036 \mathrm{~W} / \mathrm{m} \cdot \mathrm{K}$
- Densidad: $60-140 \mathrm{~kg} / \mathrm{m}^{3}$

El aislante se recubrirá exteriormente con chapa de aluminio para protegerlo de la corrosión y de humedad.

\section{Metodología de cálculo para determinar la}

\section{eficiencia energética del generador de vapor}

La eficiencia energética del generador de vapor se determinará mediante una hoja de cálculo Excel nombrada "Sistema de análisis de eficiencia para generadores de vapor", versión 2.0, elaborado por el ingeniero Pedro Rodríguez Echemendía, de la Universidad 
de Cienfuegos, Cuba, la cual se basa en la metodología de cálculo planteada por Tanquero (1987).

\section{Estimación de la dispersión de los gases contaminantes producto de la combustión}

Para la estimación de la dispersión de los contaminantes se utilizó el programa de computación Contamín, que está basado en la norma cubana (NC 39:1999, "Calidad del aire: requisitos higiénico-sanitarios"). Por medio de este programa se puede estimar la cantidad de contaminantes emitidos a la atmósfera debido al proceso de combustión.

\section{Análisis de la calidad del agua}

Para determinar la calidad del agua de alimentación de la caldera se realizarán análisis de dureza total (DT), conductividad, $\mathrm{pH}$ y concentración de cloruros, tanto al agua cruda como el agua suavizada, antes de alimentar con ella el generador de vapor. Los resultados serán comparados con las normas de calidad presentes en el laboratorio de la empresa, NRIAL 012:1992 (Minal, 1992) para aguas de este tipo.

\section{Influencia del uso de aislante en la reducción del consumo de combustible en el generador de vapor, y su posterior impacto económico}

Se evaluará el ahorro económico que se obtendría por concepto de combustible que se deja de quemar en la caldera a causa del aislamiento de las tuberías de vapor de agua con lana mineral. El costo de adquisición y montaje de este aislante fue obtenido a partir de datos suministrados por la empresa Alastor, y asciende a PC 15 el metro. El consumo anual de combustible fuel oil en la caldera fue proporcionado por el Departamento de Economía y Contabilidad de la propia fábrica, y asciende a 115721 L/año. A partir del uso de una hoja de cálculo Excel se determinarán los siguientes indicadores económicos: valor actual neto (VAN) y tasa interna de retorno (TIR) para un tiempo de 3, 6, 9, 12 y 15 meses, con respecto al ahorro económico que se obtendría por concepto de combustible dejado de quemar en la caldera a causa del aislamiento de las tuberías de vapor con lana mineral. También se calculará el periodo de recuperación de la inversión (PRI) con relación a la inversión inicial ejecutada.
Influencia del incremento de la temperatura del agua de alimentación en varios parámetros técnico-económicos de la caldera

El agua de alimentación de la caldera es suministrada a temperatura ambiente $\left(25-35^{\circ} \mathrm{C}\right)$. Sin embargo, se desea conocer el impacto que tendría el precalentamiento de esta agua hasta los $90^{\circ} \mathrm{C}$, en los siguientes parámetros técnico-económicos de la caldera:

- Consumo de combustible $(\mathrm{kg} / \mathrm{h})$

- Índice de generación de vapor ( $\mathrm{kg}$. $_{\text {vapor }} / \mathrm{kg}$. $_{\text {comb }}$ )

- Eficiencia energética de la caldera (\%)

- Cantidad de combustible ahorrado por año (t/ año) y su correspondiente resultado económico (\$/año)

Esto se efectuará empleando la hoja de cálculo Excel "Sistema de análisis de eficiencia para generadores de vapor", versión 2.0.

Impacto económico global relacionado con al aislamiento de las redes de tuberías e incremento de la temperatura del agua de alimentación

Se procederá a determinar el ahorro económico total obtenido por concepto de ahorro de consumo de combustible en el generador de vapor, al aplicar las dos variantes de mejoras propuestas, esto es: 1) aislamiento de las redes de tuberías de vapor con el aislante lana mineral, y 2) incremento de la temperatura del agua de alimentación a la caldera hasta $90{ }^{\circ} \mathrm{C}$ (precalentamiento), lo cual nos dará una visión cercana acerca de las posibilidades reales de mejoras técnico-económicas en esta sección tan importante de la fábrica.

\section{RESULTADOS Y DISCUSIÓN}

Resultados de las pérdidas de calor en las tuberías de vapor y diámetro óptimo al utilizar el aislante de lana mineral

De acuerdo con los resultados obtenidos (tabla 3), el promedio de reducción de las pérdidas de calor al ambiente al emplear el aislante de lana mineral fue de $89.97 \%$ para las tuberías horizontales, mientras que para las verticales se obtuvo un valor de $90.12 \%$, los cuales se encuentran en el rango establecido por la bibliografía consultada (Isachenko, Osipova \& Sukomel, 1973) (Fonte, Nemiróvich \& González, 1986), que es de $75-95 \%$. 
Tabla 3. Resultado de las pérdidas de calor empleando el aislante lana mineral

\begin{tabular}{|c|c|c|c|c|c|c|c|c|c|c|}
\hline \multirow[t]{2}{*}{ Tramo } & \multicolumn{2}{|c|}{$\begin{array}{l}\text { Pérdidas de calor } \\
\text { (aislada) }[\mathrm{W} / \mathrm{m}]\end{array}$} & \multicolumn{2}{|c|}{$\begin{array}{l}\text { Pérdidas de calor } \\
\text { (aislada) [W] }\end{array}$} & \multicolumn{2}{|c|}{$\begin{array}{c}\text { Reducción de } \\
\text { pérdidas de } \\
\text { calor }(\%)\end{array}$} & \multicolumn{2}{|c|}{$\begin{array}{l}\text { Espesor del } \\
\text { aislante } \\
(\mathrm{mm})\end{array}$} & \multicolumn{2}{|c|}{$\begin{array}{l}\text { Diámetro de } \\
\text { tubería con } \\
\text { aislante }(\mathrm{mm})\end{array}$} \\
\hline & $\mathbf{H}$ & V & $\mathbf{H}$ & V & $\mathbf{H}$ & V & $\mathbf{H}$ & V & $\mathbf{H}$ & V \\
\hline 1 & 47.96 & 64.80 & 592.79 & 100.44 & 89.9 & 89.9 & 12.24 & 12.39 & 84.77 & 114.00 \\
\hline 2 & 63.29 & 61.60 & 1113.90 & 86.24 & 90.0 & 90.4 & 13.11 & 13.46 & 115.12 & 116.00 \\
\hline Total & 111.25 & 126.40 & 1706.69 & 186.68 & & & & & & \\
\hline
\end{tabular}

1. Caldera-calentador de combustible

2. Caldera-disolutor

\section{Análisis de los resultados obtenidos para la eficiencia energética del generador de vapor}

En la figura 2 y la tabla 4 se muestran los resultados obtenidos al aplicar la metodología de cálculo seleccionada para determinar la eficiencia energética del generador de vapor, mientras que en la figura 3 se muestra el diagrama de Sankey obtenido.

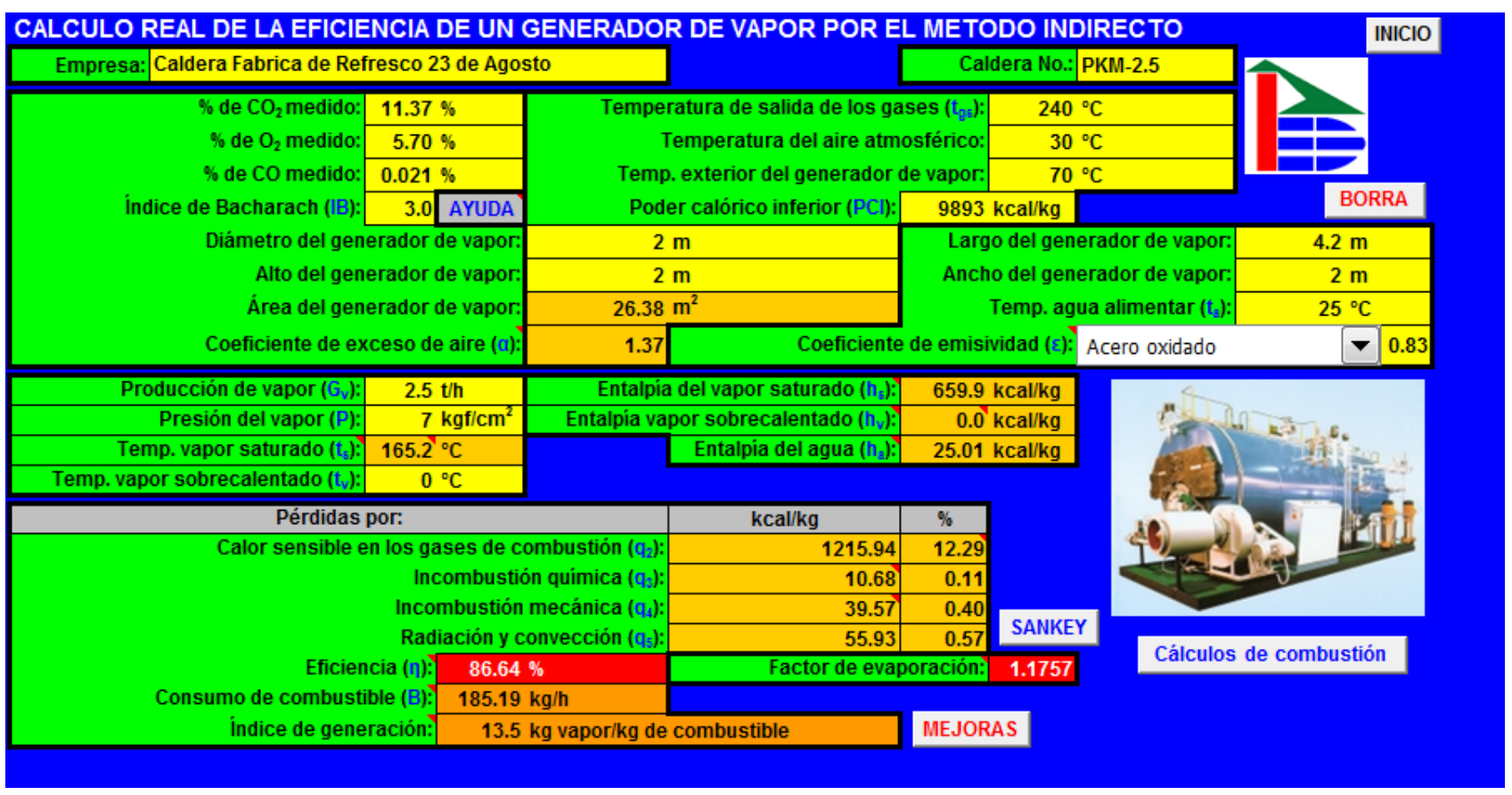

Figura 2. Resultados obtenidos al evaluar la eficiencia energética de la caldera

Según se puede observar en la tabla 4, la eficiencia energética tuvo un valor de $86.85 \%$. Este resultado se encuentra dentro del rango recomendado (75-88 \%) por la bibliografía consultada (Colciencias, 2009) (Morales, 2011) para una caldera de este tipo. Entre las posibles causas que posibilitan la reducción de la eficiencia energética de una caldera se encuentran, según Krishnanunni et al. (2012) y Morales (2011):

- Tratamiento deficiente o no adecuado del agua de alimentación, lo cual origina la formación de depo- 


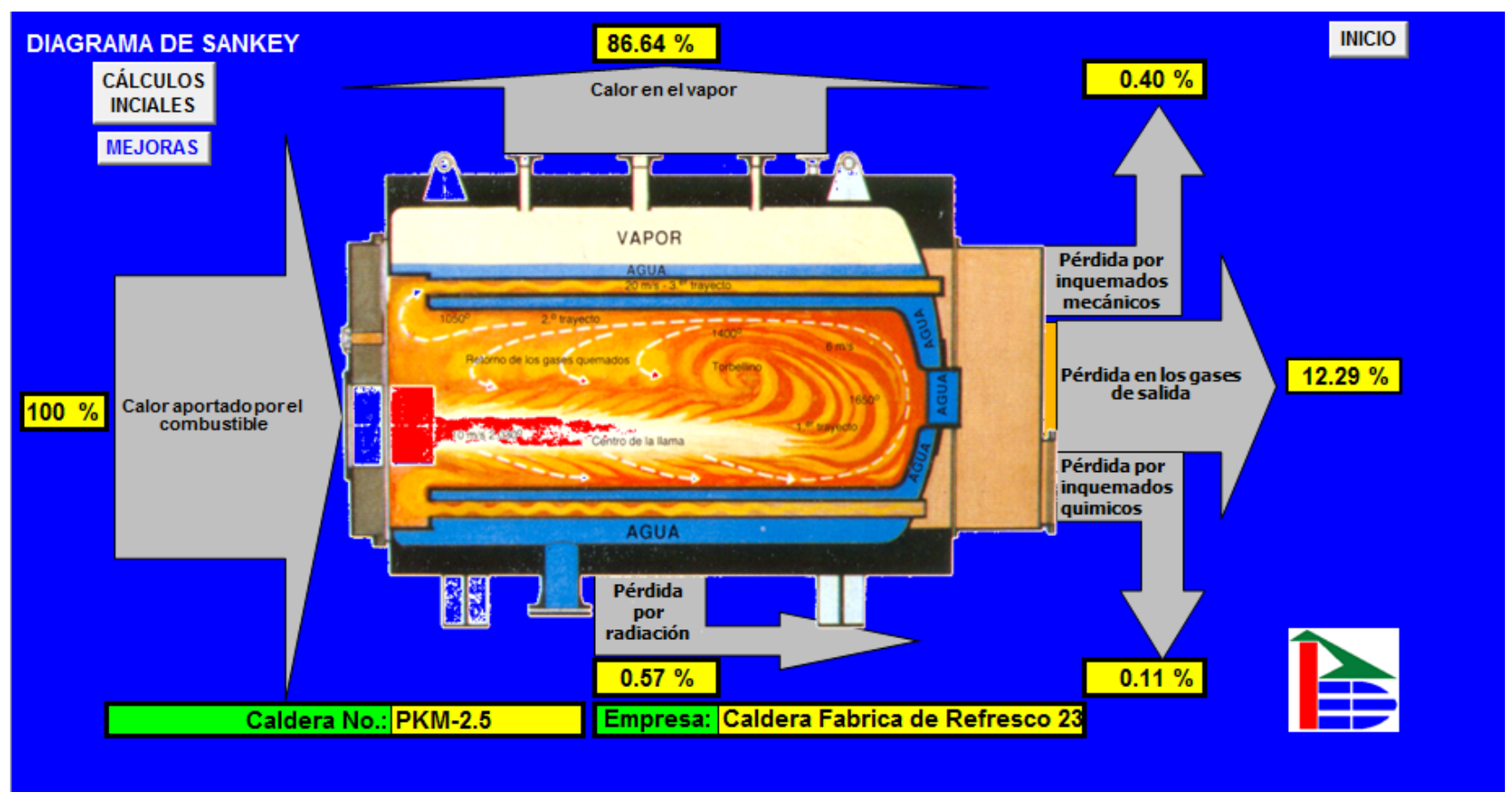

Figura 3. Diagrama de Sankey de la caldera evaluada

Tabla 4. Resultados obtenidos en cuanto a la eficiencia energética de la caldera

\begin{tabular}{ccc}
\hline Parámetro & Valor & Unidades \\
\hline Pérdidas de calor sensible en los gases de combustión & 12.29 & $\%$ \\
\hline Pérdidas por incombustión química & 0.11 & $\%$ \\
\hline Pérdidas por incombustión mecánica & 0.40 & $\%$ \\
\hline Pérdidas por convección y radiación & 0.57 & $\%$ \\
\hline Consumo de combustible & 185.19 & $\mathrm{~kg}_{{ }_{\text {comb }}} / \mathrm{h}$ \\
\hline Índice de generación & 13.5 & $\mathrm{~kg}_{\text {vap }} / \mathrm{kg}_{\text {comb }}$ \\
\hline Factor de evaporación & 1.1757 & - \\
\hline Coeficiente de exceso de aire & 1.37 & - \\
\hline Eficiencia energética & 86.64 & $\%$ \\
\hline
\end{tabular}

siciones en la superficie de intercambio de calor interna de la caldera (tubos), reduciéndose así la eficiencia de traspaso (transferencia) de calor desde los gases de combustión hacia el agua, con el consiguiente aumento del consumo de combustible.

- Sistema de combustión deficiente y baja calidad del combustible empleado (presencia de un gran número de impurezas), lo cual origina que no se genere el calor requerido por el proceso, producto de la combustión incompleta del combustible.

- Aislamiento deficiente del generador de vapor.
- Ausencia de precalentamiento del agua de alimentación y/o del aire de combustión.

- Circulación deficiente de los gases de combustión y/o vapor de agua en el interior de la caldera, producto de un diseño mecánico incorrecto de la misma, es decir, el equipo puede estar sub o sobredimensionado con relación a la capacidad real de producción de vapor.

La temperatura de los gases de combustión es de $240{ }^{\circ} \mathrm{C}$, la cual se encuentra 1.33 veces por encima de la reportada en la literatura técnica (Colciencias, 
2009) (Faires, 1991) para una caldera de este tipo (180 $\left.{ }^{\circ} \mathrm{C}\right)$. Las siguientes son, fundamentalmente, las razones:

- Ensuciamiento de los tubos de intercambio de calor. La combustión es incompleta y el arrastre de partículas (dependiendo de la calidad del combustible utilizado) puede ir ensuciando poco a poco los tubos.

- Dosificación incorrecta del combustible. Otra causa puede ser una mezcla incorrecta del combustible y comburente, lo cual produce mayor temperatura de la llama y los humos. Esto se debe fundamentalmente a un mal estado técnico del quemador.

- Velocidad excesiva de los gases de combustión (humos). Una velocidad de salida excesivamente alta no permite la permanencia de los humos calientes el tiempo suficiente para ceder la energía térmica que transportan.

Se obtuvo un valor del consumo de combustible de $184.74 \mathrm{~kg} / \mathrm{h}$, mientras que el índice de generación de vapor (IGV) fue de $13.533 \mathrm{~kg}$ de vapor $/ \mathrm{kg}$ de combustible.

Al comparar los resultados obtenidos de las pérdidas energéticas (tabla 4), con aquellos reportados por la literatura especializada (Colciencias, 2009) (Faires, 1991), se puede considerar lo siguiente:

- Las pérdidas de calor en los gases de combustión $(12.29 \%)$ se encuentran dentro del rango establecido (6-30\%).
- Las pérdidas por incombustión química (0.11\%) se hallan también dentro del intervalo recomendado (0-5\%)

- Las pérdidas por incombustión mecánica se encuentran cercanas a cero $(0.40 \%)$, cuando se sugiere que deben ser de $0 \%$

- Las pérdidas por radiación y convección $(0.35 \%)$ se encuentra por debajo del rango permitido (2-6\%), lo cual indica un aislamiento térmico eficiente del generador de vapor

\section{Estimación de las emisiones de los compuestos contaminantes producto de la combustión del combustible}

En la figura 4 se muestra la ventana principal del software Contamín, en la cual se detallan los diferentes valores que deberán tener los parámetros considerados para que este programa funcione, mientras que en la figura 5 se observa el resultado de concentración obtenido para el compuesto dióxido de carbono $\left(\mathrm{CO}_{2}\right)$.

La tabla 5 muestra las concentraciones de cada compuesto contaminante generado durante la combustión del fuel oil en la caldera $\left(\mathrm{CO}_{2}, \mathrm{CO}, \mathrm{SO}_{2}, \mathrm{NO}_{2}\right.$, material particulado y $\mathrm{CH}_{4}$ ), las cuales fueron determinadas mediante el software Contamín, y su comparación con los valores límites establecidos por la norma cubana vigente (ONN, 2010).

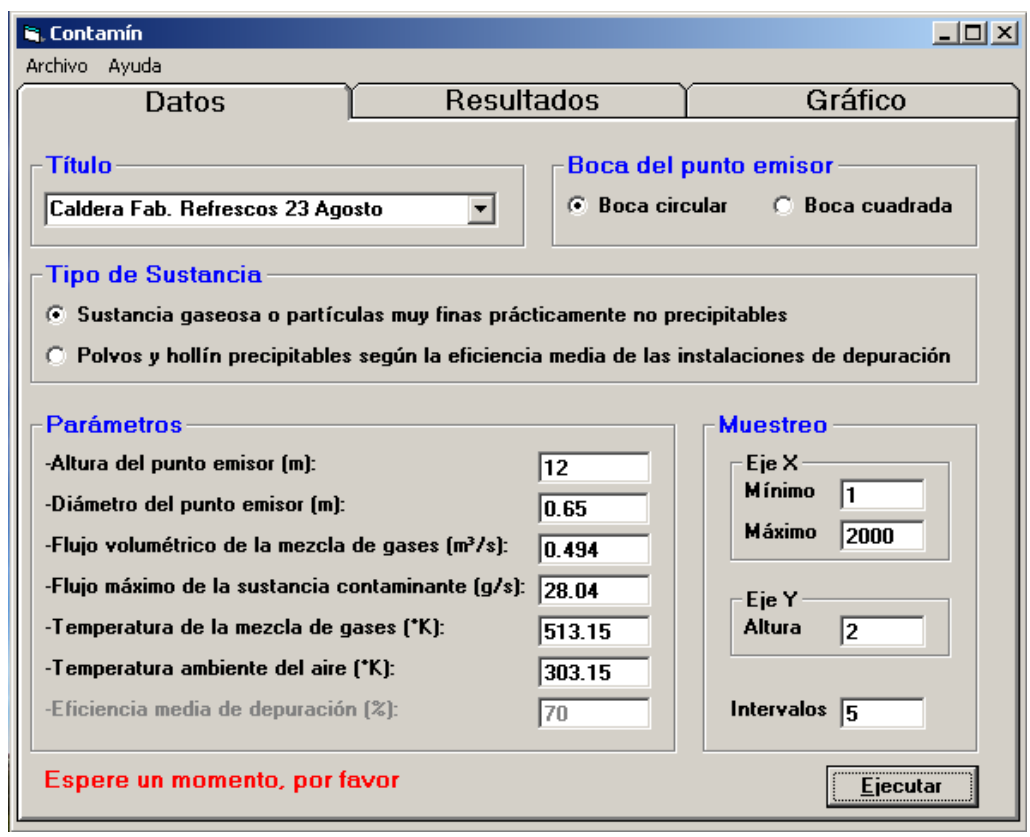

Figura 4. Ventana principal del software Contamín 


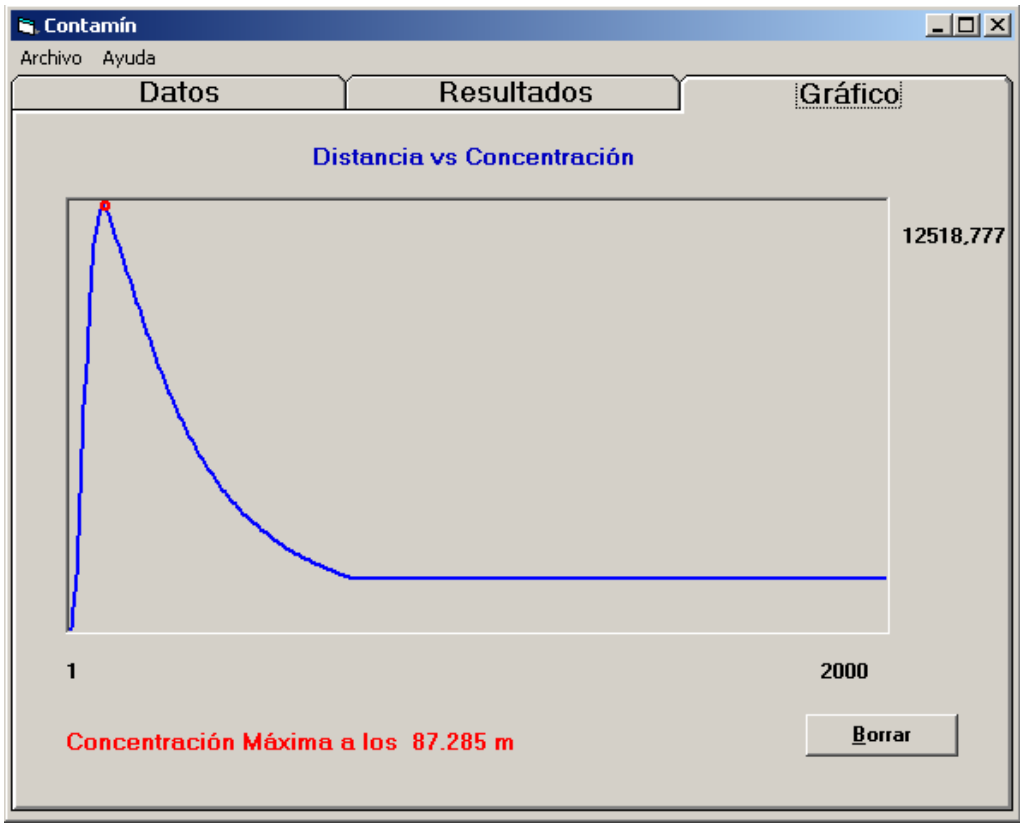

Figura 5. Concentración máxima obtenida para el compuesto $\mathrm{CO}_{2}$

Tabla 5. Resultados obtenidos de las emisiones de gases contaminantes mediante el Contamín y su comparación con la norma cubana vigente

\begin{tabular}{cccc}
\hline Compuesto & $\begin{array}{c}\text { Concentración calculada } \\
\left(\mathbf{m g} / \mathbf{m}^{3}\right)\end{array}$ & $\begin{array}{c}\text { Valor según norma } \\
\left(\mathbf{m g} / \mathbf{m}^{3}\right)\end{array}$ & ¿Cumple? \\
\hline $\mathrm{CO}_{2}$ & 12.52 & - & - \\
$\mathrm{SO}_{2}$ & 1.97 & 5 & Sí \\
$\mathrm{NO}_{x}$ & 0.0356 & 700 & Sí \\
Material particulado & 0.00254 & - & - \\
$\mathrm{CH}_{4}$ & 0.0204 & 50 & Sí \\
\hline
\end{tabular}

Según la norma consultada, el compuesto dióxido de azufre $\left(\mathrm{SO}_{2}\right)$ debe tener una emisión máxima admisible de $5 \mathrm{mg} / \mathrm{Nm}^{3}$ para una caldera que consume fuel oil. El valor de emisión obtenido para este compuesto por medio del software Contamín fue de $1.97 \mathrm{mg} /$ $\mathrm{Nm}^{3}$, que, según se puede observar, se encuentra por debajo del valor establecido. En el caso del material particulado (MP), la emisión máxima no deberá exceder los $50 \mathrm{mg} / \mathrm{Nm}^{3}$, mientras que el valor calculado de $\mathrm{MP}$ en el Contamín fue de $0.0204 \mathrm{mg} / \mathrm{Nm}^{3}$, que también es menor que el máximo establecido por las normas cubanas. Por último, para los óxidos de nitrógeno $\left(\mathrm{NO}_{\mathrm{x}}\right)$, el valor calculado a través del Contamín fue de $0.0356 \mathrm{mg} / \mathrm{Nm}^{3}$, que también se encuentra por debajo del valor máximo normado $\left(700 \mathrm{mg} / \mathrm{Nm}^{3}\right)$.

Finalmente, según el programa Contamín, la distancia (radio) a la cual se alcanza la mayor concentración de contaminantes es de $81.384 \mathrm{~m}$, que se encuentra por encima del radio mínimo admisible según la norma cubana consultada (ONN, 2010), que es de 50 metros. Esto significa que los gases contaminantes generados afectan tanto el medioambiente del interior de la fábrica como las zonas adyacentes a esta.

\section{Evaluación de los resultados de los ensayos de calidad realizados tanto para el agua cruda como para la suave}

Con relación a los resultados obtenidos de los análisis físico-químicos efectuados tanto al agua cruda como a la suave, se obtuvieron los valores expuestos en la tabla 6. 
Tabla 6. Resultados de los análisis físico-químicos efectuados tanto al agua cruda como a la suave, y su comparación con la norma cubana vigente

\begin{tabular}{ccccc}
\hline Parámetro & Agua cruda & Agua suave & Norma (agua suave) & $\begin{array}{c}\text { ¿Cumple con la norma } \\
\text { NRIAL 012:1992? }\end{array}$ \\
\hline Dureza total $(\mathrm{ppm})$ & 127.00 & 0.15 & $0-10$ & Sí \\
$\mathrm{pH}$ & 7.78 & 7.46 & $7.0-9.0$ & Sí \\
Conductividad & 394.5 & 410 & - & - \\
$\begin{array}{c}\text { Concentración cloruros } \\
(\mathrm{ppm})\end{array}$ & 162 & 105 & Igual o menor & Sí \\
\hline
\end{tabular}

De forma general, se puede concluir que el sistema de tratamiento del agua con que se alimenta la caldera es eficiente, ya que se obtiene un agua suave que cumple con todas las parámetros físico-químicos referidos en la norma NRIAL 012:1992 (Minal, 1992) para un agua de este tipo.

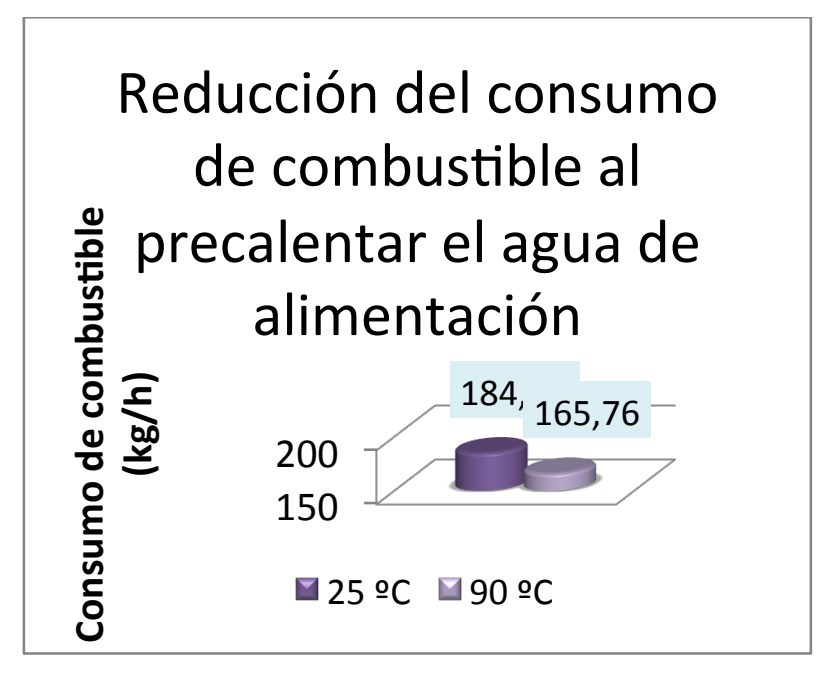

\section{Análisis de las alternativas de mejoras}

Precalentamiento del agua de alimentación a la caldera desde $25{ }^{\circ} \mathrm{C}$ hasta $90{ }^{\circ} \mathrm{C}$

El aumento de la temperatura del agua de alimenta-

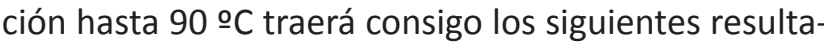
dos (véase la figura 6):

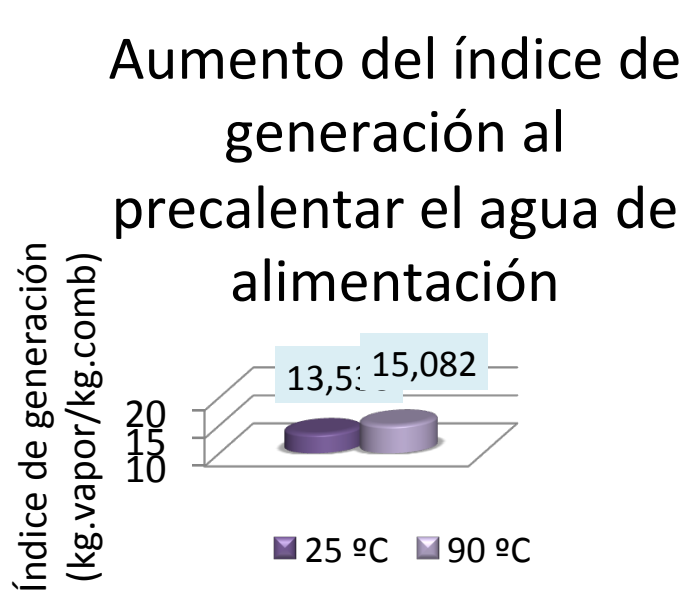

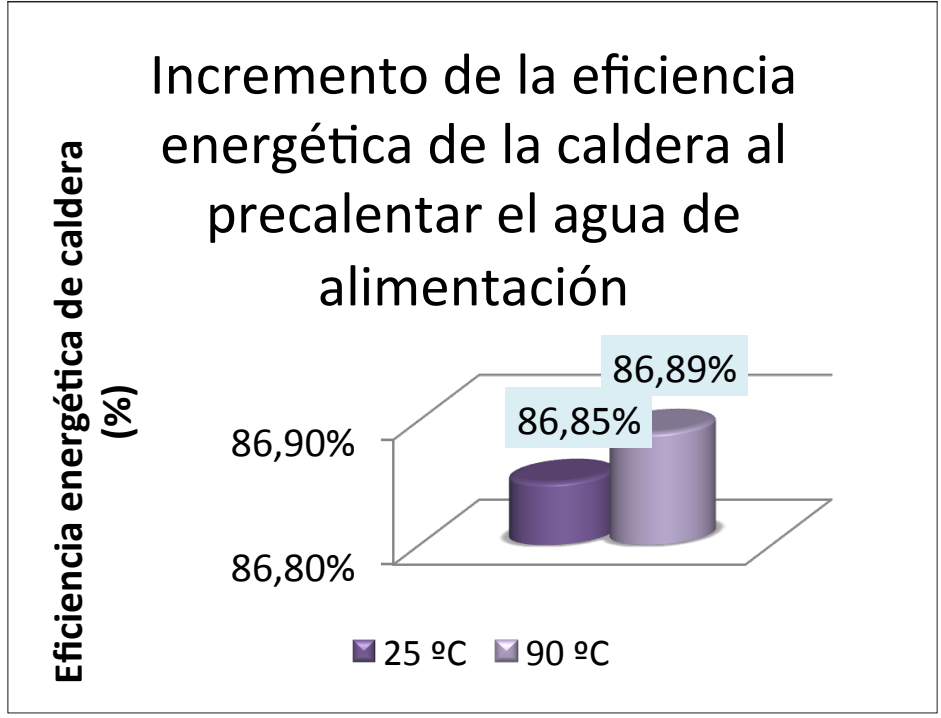

Figura 6. Resultados obtenidos al precalentar el agua de alimentación a la caldera desde $25^{\circ} \mathrm{C}$ hasta $90^{\circ} \mathrm{C}$ 
- Ahorro de $19.34 \mathrm{~kg} / \mathrm{h}$ de combustible

- Incremento del índice de generación de vapor en $1,574 \mathrm{~kg} \cdot{ }_{\text {vapor }} / \mathrm{kg}$. $_{\text {comb }}$

- Incremento de la eficiencia energética de la caldera en un $0.20 \%$

Todo ello traerá consigo que se ahorren 17.41 toneladas $(17410 \mathrm{~kg}$ ) de fuel oil al año. Tomando en cuenta que el litro de fuel oil cuesta PC 0.5039, según datos suministrados por el Departamento de Contabilidad de la propia fábrica, y que la densidad de este combustible es de $0.966 \mathrm{~kg} / \mathrm{L}$ aproximadamente (McCain, 1990), el precalentamiento del agua de alimentación le permitirá ahorrar a la empresa PC 9081.70 por año, por concepto de combustible que no ha alcanzado a consumirse en el generador de vapor.

\section{Aislamiento de las tuberías de vapor con lana mineral}

El aislamiento de los tramos de tuberías por donde circula vapor traerá consigo los siguientes ahorros:

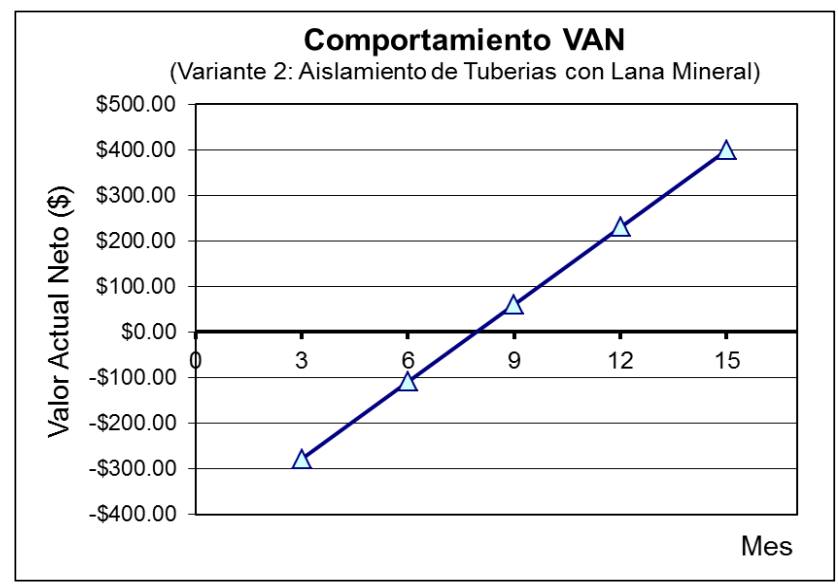

- Combustible ahorrado: $1628.73 \mathrm{~kg}_{\text {comb }} /$ año ( 1.6 t/año)

- Ahorro económico por combustible ahorrado: PC 850.22/año

Con relación a los datos obtenidos durante el levantamiento hidráulico efectuado en la fábrica, en la actualidad existen $32.91 \mathrm{~m}(\sim 33 \mathrm{~m})$ de tuberías sin aislar, entre horizontales y verticales. El aislamiento de todos estos tramos con lana mineral significaría un costo total de PC 523.66, tomando en cuenta un $2 \%$ de sobrecosto por concepto de montaje. Considerando que se ahorran alrededor de PC 77.29 al mes por concepto de combustible que no ha alcanzado a consumirse en la caldera, el periodo de retorno de la inversión (PRI) será de 122 días, mientras que el valor actual neto (VAN) y la tasa interna de retorno (TIR) para un tiempo de 12 meses ( 1 año), tendrán valores de PC 229.96 y $10 \%$, respectivamente, tomando en cuenta una tasa de interés del $17 \%$. En la figura 7 se muestra el comportamiento de las variables VAN y TIR con respecto al tiempo $(3,6,9,12$ y 15 meses).

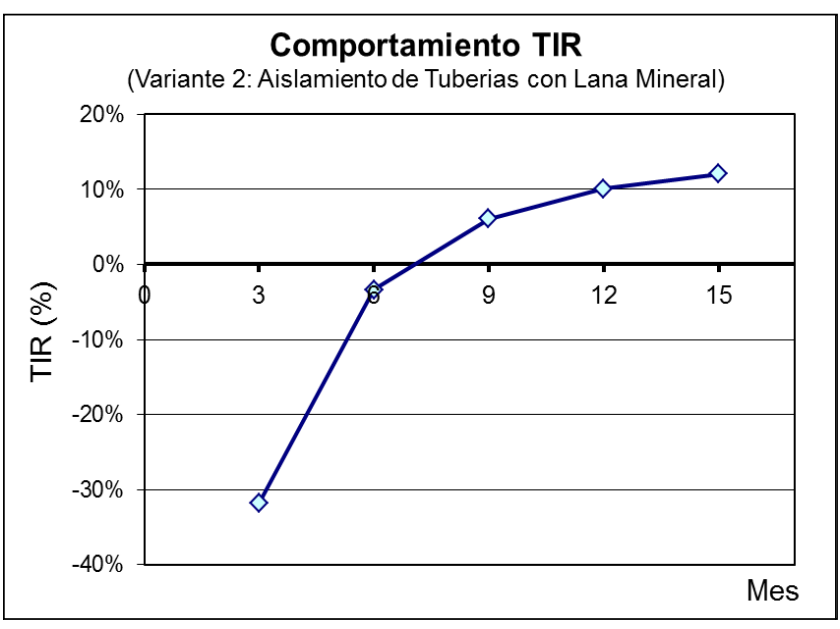

Figura 7. Comportamiento de las variables VAN y TIR con respecto al tiempo para la variante de mejora $n .^{\circ} 2$,

"Aislamiento de las tuberías con lana mineral"

\section{Ahorro económico total}

El ahorro económico total que se obtendrá por año al aplicarse ambas variantes de mejoras será de

$\$ 9081.7+\$ 850.22=\$ 9931.92 /$ año. 


\section{CONCLUSIONES}

1. La distancia a la cual se obtiene la concentración máxima para cada contaminante gaseoso es de $87.285 \mathrm{~m}$, la cual se encuentra por encima de la norma cubana establecida (50 m).

2. Se obtiene un agua suavizada con una dureza total cercana a cero $(0.15 \mathrm{ppm})$, lo cual es indicativo de una eficiente operación del sistema de tratamiento de agua.

3. El empleo del aislante de lana mineral reduce las pérdidas de calor en las tuberías horizontales en un $89.97 \%$, y en las tuberías verticales en un $90.12 \%$.

4. La eficiencia energética de la caldera es de $86.64 \%$.

5. La inversión total del aislante de lana mineral será de PC 523.66, y se recuperará en 122 días.

6. El incremento de la temperatura del agua con que se alimenta el generador de vapor hasta $90^{\circ} \mathrm{C}$ traerá consigo un ahorro económico anual de PC 9087.10.

7. El aislamiento de los $32.91 \mathrm{~m}$ de tuberías de vapor con lana mineral traerá consigo un ahorro económico anual de PC 850.22 por concepto de combustible que no alcanza a quemarse en la caldera.

8. La aplicación de las dos variantes de mejora propuestas en el sistema termoenergético de la fábrica traerá consigo un ahorro total anual de PC 9931.92.

\section{REFERENCIAS}

Calorcol (2014). Catálogo general de productos (pp. 4-12). Antioquia: Calorcol.

Colciencias (2009). Eficiencia energética en la generación y distribución de vapor (Informe n. ${ }^{\circ}$ 112-2009). Medellín: Universidad del Atlántico y Universidad Autónoma de Occidente.

Faires, V. W. (1991). Termodinámica. La Habana: Editorial Científico-Técnica.

Fonte, A., Nemiróvich, P. M., y González, R. (1986). Cálculo de las pérdidas de calor y del espesor del aislante (pp. 10-22). Camagüey: Editorial Universidad de Camagüey.

Guevara, R. F. (2013). Curso plantas consumidoras de energía. Separata II-generadores de vapor pirotubulares (pp. 13-29). Nuevo Chimbote: Universidad Nacional del Santa.

Isachenko, V., Osipova, V., y Sukomel, A. (1973). Transmisión del calor (pp. 39-67). Bilbao: Editores Marcombo.

Isover (2012). Manual de aislamiento en la industria (pp. 3-8), Badalona: Isover.

Kasatkin, A. G. (1985). Operaciones básicas y aparatos en la tecnología química (pp. 12-40). La Habana: Pueblo y Educación.

Kern, D. Q. (1999). Procesos de transferencia de calor (pp. 76-212). México D. F.: Compañía Editorial Continental.

Krishnanunni, S., Paul, J., Potti, M., \& Mathew, E. M. (2012). Evaluation of Heat Losses in Fire Tube Boiler: International Journal of Emerging Technology and Advanced Engineering, 2(12), 301305.

López, A. (2009). Evaluación del generador de vapor de la planta piloto del centro de estudios anticorrosivos y tensoactivos de la Universidad de Matanzas. Monografías, 2(4), 1-15.

McCain, W. D. (1990). The Properties of Petroleum Fluids (pp. 112-136). Oklahoma: PennWell Books.

Meena, V. S., \& Singh, M. P. (2015). Efficiency Assessment and Improvement of Boiler at Super Thermal Power Station: International Journal for Advance Research in Engineering and Technology, 3(4), 32-36.

Mijéev, M. A., y Mijeeva, I. M. (1979). Fundamentos de termotransferencia (pp. 39-58). Moscú: Editorial Mir.

Minal (1992). NRIAL 012: Especificaciones de calidad. Aguas industriales-aguas de calderas. La Habana: Minal.

Morales, E. F. (2011). Calderas (pp. 10-68). Chile: Universidad Austral de Chile. 
Oficina Nacional de Normalización (2010). NC-TS 803:2010. Calidad del aire: Emisiones máximas admisibles de contaminantes a la atmósfera en fuentes fijas puntuales en instalaciones generadoras de electricidad y vapor. La Habana: Oficina Nacional de Normalización.

Pankrátov, G. (1987). Problemas de termotecnia (pp. 30-94). Moscú: Editorial Mir.

Pérez, L. (1972). Generadores de vapor (pp. 90-136). Ciudad de La Habana: Pueblo y Educación.
Raut, S., Kumbhare, S. B., \& Thakur, K. C. (2014). Energy Performance Assessment of Boiler at P.S.S.K. Ltd, Basmathnagar, Maharashtra State. International Journal of Emerging Technology and Advanced Engineering, 4(12), 242-253.

Tanquero, N. (1987). Guía metodológica para proyectos de curso de generadores de vapor (pp. 28-96). Ciudad de La Habana: Pueblo y Educación. 